\title{
Horizons/Théâtre
}

Revue d'études théâtrales

$14 \mid 2019$

Les arts du spectacle dans l'Afrique subsaharienne - 2

\section{La crise de la doxa esthétique ou contre les frontières dans le théâtre de José Pliya}

\section{Bassidiki Kamagate}

\section{(2) OpenEdition}

1 Journals

\section{Édition électronique}

URL : https://journals.openedition.org/ht/1464

DOI : $10.4000 /$ ht. 1464

ISSN : 2678-5420

Éditeur

Presses universitaires de Bordeaux

\section{Édition imprimée}

Date de publication : 1 janvier 2019

Pagination : 60-73

ISBN : 979-10-300-0718-3

ISSN : 2261-4591

\section{Référence électronique}

Bassidiki Kamagate, "La crise de la doxa esthétique ou contre les frontières dans le théâtre de José Pliya », Horizons/Théâtre [En ligne], 14 | 2019, mis en ligne le 01 juin 2022, consulté le 30 juin 2022.

URL : http://journals.openedition.org/ht/1464 ; DOI : https://doi.org/10.4000/ht.1464

\section{(c) $(1) \&$}

La revue Horizons/Théâtre est mise à disposition selon les termes de la Licence Creative Commons Attribution - Pas d'Utilisation Commerciale - Pas de Modification 4.0 International. 


\section{BASSIDIKI KAMAGATE}

Chercheur associé au groupe d'études et de recherches FRED EA 6311 (Limoges, France), Bassidiki Kamagate est maître de conférences à l'Université Alassane Ouattara où il enseigne le théâtre. Il est l'auteur de nombreuses publications portant sur l'éthique et l'esthétique du théâtre négro-africain. Parmi celles-ci, on retiendra : "Les recluses de Koffi Kwahulé : dramaturgie et sociocritique de l'Afrique contemporaine ", in @nalyses(Ottawa, Canada), 2013 ; "L'impolitesse dans Bintou de Koffi Kwahule : de la désinvolture sociale à la pièce-parabole » dans MuloFarenkia Bernard (Dir.), Impolitesse et rituels interactionnels en contextes plurilingues et multiculturels, Moosstrasse 1, Peter Lang - International Academic ; Publishers, 2016; Bidy Cyprien Bodo, Moussa Coulibaly et Bassidiki Kamagaté, Les écritures de l'horreur en littératures africaines, Paris, L'Harmattan, 2016.

Mail: baskamag@gmail.com

Résumé : La poétique dramatique de José Pliya déjoue les horizons d'attente du lecteur-spectateur nourri de préjugés doctrinaux à l'égard de l'art théâtral négro-africain. La contestation des idées reçues et le dévoiement des a priori esthétiques fondent le théâtre de Pliya.

L'écriture dramatique de cet auteur convoque tous les possibles, notamment le refus du

Abstract : José Pliya's theatrical art is marked by a form of poetic and aesthetic disaffiliation with the existing theater. His dramatic writing thwarts the horizons of expectation because it is marked by the seal of the renewal of dramatic instances. Without rejecting the dramatic form, he reinvents it by cloisonnement générique, le déploiement des richesses de l'intertextualité, le mélange des arts. La dramaturgie de Pliya renferme divers procédés de violation des frontières artistiques que le présent travail envisage décrypter les modes et les valeurs.

Mots-clés : doxa esthétique, mutation, transtextualité, dialogisme artistique, théâtre tout-monde.

enlarging it. Heterogeneous textual materials now express the vitality of African dramatic art, which was forced to adjust to changes.

Keywords: aesthetic norms, mutation, transtextuality, dialogism, artistic, all world theater. 


\section{La crise de la doxa esthétique ou contre les frontières dans le théâtre de José Pliya}

\section{Introduction}

L'acte fondateur de L'ÉCRIture dramatique de José Pliya est la pièce Konda le roquet. Il y confesse :

Ma pièce arrive à terme. Vos mines réjouies me laissent deviner que vous n'en êtes pas mécontents et vous devez me croire un auteur comblé. Il n'en est rien. Mon drame est total car l'auteur de kondo le requin, c'est mon père. Pour que mon cuvre prenne vie, il me fallait tuer celle du père. Abominable mais indispensable crime. (Acte I, Sc. V, p. 99)

Le parricide inféré dans la mise en abyme de Kondo le requin laisse entrevoir la rupture de Pliya avec les codes et les conventions à l'œuvre dans les créations du père. Ainsi, pour Stéphanie Bérard, « la théâtralité de Konda le roquet, à travers le jeu de l'emboîtement du théâtre dans le théâtre, permet d'interroger la création théâtrale, le pouvoir de l'illusion et l'avenir des dramaturgies africaines ${ }^{1} \gg$. Le sacrilège du parricide clame une désaffiliation artistique renvoyant à un renouveau de l'esthétique dramaturgique dont son œuvre déploie les modalités, notamment par son insoumission au déterminisme des origines. La parodie du titre formule cette envie de libération des marques ethniques tout en figurant un désir de novation signifié par la mutation $\mathrm{du} \ll \mathrm{o} \gg$ en $\ll \mathrm{a} \gg$. Ceci constitue une évidence, selon Bérard qui soutient que « la substitution vocalique et animalière - du o en a dans le passage de Kondo le requin à Konda le roquet - annonce de manière burlesque le renouveau du théâtre africain, o et a étant l'oméga et l'alpha ${ }^{2} \gg$. Aussi, la dangerosité du requin s'est muée en aboiements dérisoires afin de traduire la nécessité de la revitalisation.

Dès lors, la poétique dramatique de José Pliya déjoue les horizons d'attente du lecteur-spectateur nourri de préjugés doctrinaux à l'égard de l'art théâtral négro-africain. La contestation des idées reçues et le dévoiement de l'a priori esthétique fondent son théâtre. L'écriture dramatique convoque tous les possibles, notamment le refus du cloisonnement générique, le déploiement des richesses de l'intertextualité, le mélange des arts. La dramaturgie de Pliya 
renferme divers procédés de violation des frontières artistiques dont les modes et les valeurs seront, ici, déclinés.

\section{Le théâtre de José Pliya, une odyssée moderne}

L'œuvre théâtrale de José Pliya échappe aux frontières physiques et psychologiques de l'identité ethnique d'une écriture. Elle procède d'un périple à travers l'histoire de toutes les sociétés humaines. Le principe de la mobilité créatrice qui prévaut dans son théâtre trouve son fondement dans la pièce Nègrerrances. Au-delà de la migration et de l'exil induit dans ce titre, l'auteur énonce l'altérité et l'universalité qui sous-tendent son œuvre transie d'ouverture. Le Balayeur peut alors inciter Nicolas au nomadisme, au voyage pédagogique : «Il te faut étreindre la terre pour entrer en connaissance. » (Nègrerrances, II, p. 35) De la connaissance de l'autre surgit la connaissance de soi. Pliya enseigne ainsi que la terre est la patrie commune à tous les hommes. Il sanctionne les frontières, marqueuses de l'infâme escalade identitaire.

\section{Les mots de tous les possibles}

Entorse ou enrichissement de la langue française, certains titres de pièces de José Pliya accrochent le lecteur-spectateur par leur forme lexicale insolite. Ce sont Nègrerrances et Les Effracteurs. Les deux substantifs relèvent de ce que Catherine Fromilhague nomme mots-valises, ces néologismes résultant de la « combinaison et (de la) fusion d'au moins deux termes partiellement homophones $^{3} \gg$. En lexicologie synchronique, la fusion, en tant que procédé de formation lexicale, est l'union intégrale, l'enchâssement unifiant d'au moins deux mots pour en faire un. Dans ce procédé, un des mots apparaît tronqué voire même méconnaissable.

Partant, il ressort que «nègrerrances » naît de l'agglutination des substantifs «nègre » et « errances » afin de traduire l'incessante violation des frontières par le nègre, qui se moque des limites territoriales pour devenir un citoyen du monde. Ici, «nègrerrances » est le fruit d'une haplologie : la syllabe-voyelle /e/constitue à la fois la fin du mot «nègre » et le début du mot « errance. »

Le mot «effracteurs », quant à lui, résulte d'une dérivation propre. Ici, l'auteur part de la base lexicale / - fract- / qui bénéficie déjà du préfixe /ef-/ et lui adjoint un suffixe nominale /-eur/pour désigner l'auteur de l'effraction. Sont ainsi génériquement désignées, dans la pièce de Pliya, toutes ces personnes qui commettent des effractions, c'est-à-dire qui opèrent des ruptures, des forcements ou des enlèvements de tout dispositif servant à fermer un 
passage ou une clôture. On peut encore soupçonner le mot « effracteurs » d'être né de l'haplologie des mots « effraction » et «acteurs ». En tout état de cause, ce néologisme lexical décline la trame même de la pièce : la mise en scène des acteurs d'une effraction.

En procédant ainsi par invention lexicale par haplologie, il happe les frontières qui séparent les mots dans leur existence isolée, pour les rapprocher dans une intimité parfaite qui vient donner congé à l'étanchéité des frontières antérieures. C'est un marqueur symbolique qui charrie le projet de José Pliya de concevoir une sorte de troisième être qui surmonte les identités rhizomes des composantes de son origine. Les mots inventés se lisent alors comme une allégorie de la trans-ethnicité comme dissolution des frontières raciales. La modification des mots induit une dislocation, une déconstruction de la dualité AUTRE vs MOI. Les frontières fonctionnent comme des « schèmes cognitifs, émotionnels, symboliques ${ }^{4} \gg$ participant d'un certain tribalisme ainsi condamné par Pliya.

Les frontières ne sont que des inepties nécessaires à ceux qui veulent se fabriquer des ennemis. Dans Nous étions assis sur les rivages $d u$ monde..., L'Homme gifle La Femme dont il refuse les compliments à cause de leurs différences devenues différends.

La Femme: Vous permettez que je vous fasse un compliment?

L'Homme :...

La Femme: Vous êtes beau.

L'Homme :...

$[\ldots]$

La Femme : Expliquez-moi vos raisons.

L'Homme :...

La Femme: Je vous écoute.

Il la gifle violemment. Elle tombe à terre, à genoux, à ses pieds.

L’Homme : Vous avez compris ? (p. 83-84)

Le geste de L'Homme prouve que les frontières, tout comme les identités, constituent des menaces à la paix sociale. Elles créent « un autre soi-même qu'il faut altériser, noircir, et rendre menaçant, afin que l'usage de la violence puisse apparaître légitime ${ }^{5} \gg$. La nécessité de la suppression des frontières 
aussi bien identitaires que matérielles s'impose afin d'instaurer l'harmonie sociale.

\section{Pour un passage des frontières culturelles et historiques : la transtextualité}

La transtextualité se conçoit comme le dévoilement des réminiscences d'autres textes dans un texte donné. Pour Gérard Genette, il s'agit de « tout ce qui le met en relation, manifeste ou secrète, avec d'autres textes ${ }^{6} \gg$. Les œuvres dramatiques de Pliya sont investies d'occurrences culturelles et historiques qui confirment la poétique de la transtextualité qui les élève au-dessus des cloisons territoriales et identitaires.

L'enthousiasme de Pliya pour la disparition des frontières se traduit par l'intertextualité, un élément essentiel de la poétique de ses pièces. La décomplexion culturelle autorise l'écriture de la pièce Le complexe de Thénardier', dont le tribut au roman Les misérables de Victor Hugo s'infère dans le nom Thénardier. La dynamique dramatique de cette pièce emprunte au drame bourgeois les dissensions maître/valet. Mais ici, Vido - la fille de ménage ne rabaisse pas le maître (La Mère) par sa malice et ses traits d'esprit comme Figaro dans Le mariage de Figaro de Beaumarchais. Le complexe de Thénardier conçoit le drame de la condition du maître devenu dépendant du travail du valet. Aucune inversion ou renversement de situation qui fait du maître le valet de son valet. Le nom Thénardier se présente comme une citation, expression de la fascination de l'auteur pour le roman d'Hugo. Le rapport de servitude qui unit Vido à La Mère reprend celui des Thénardier et de Colette. La substitution des Thénardier par La Mère et celle de Colette par Vido convoque le réinvestissement positif de l'hypotexte dans l'hypertexte. Ceci procède de, selon Georges-Élia Sarfati, « la réitération d'un geste, par la conformité sérieuse à des modèles invoqués pour la légitimité qu'ils confèrent au discours qui s'en inspire et dont, en retour, le "nouveau" discours actualise la légitimité $^{8} \gg$. Pliya ne ressasse pas Les misérables, il le sous-entend.

L'errance socio-culturelle se ressent, aussi, dans Les Effracteurs, pièce inspirée sans doute de la saga de la cavale de Boni et Clyde (Bonie et Clive), fugitifs célèbres des faits divers des États-Unis. Leur amour est ici perturbé par l'intrusion, dans leur repaire, de cambrioleurs surpris de leur présence dans cette maison supposée vide de ses propriétaires. Dans le cas de cette pièce, l'intention de disqualification de l'actualité criminelle se décline dans le sous-titre «bouffonnerie criminelle ». Il se lit comme une alliance des contraires, un oxymore semblable à un commentaire des actes criminels dont les auteurs se trouvent déchus de la gloriole qui accompagne bien souvent 
le récit de leurs différents forfaits. L'auteur parvient à construire la modalité d'une certaine intermédialité en appliquant à un événement médiatique l'un des traits du registre comique : la bouffonnerie. Cette intertextualité est implicite, relative. La réticence face à une telle lecture s'efface avec le parallélisme de construction qui s'établit entre «bouffonnerie criminelle» et le titre de certaines émissions judiciaires télé dont « chroniques criminelles ».

Ainsi, la Seconde Guerre mondiale constitue la trame d'Une famille ordinaire ${ }^{9}$. Puisque les frontières n'existent pas, l'histoire du nazisme allemand est l'histoire de toute l'humanité comme l'atteste d'ailleurs l'implication de la quasi-totalité des pays dans le conflit. C'est cela qui rend caduques les critiques qui reprochent à l'auteur d'avoir ignoré le génocide rwandais, lui l'Africain. Par transfert, rétorque Pliya, l'extrémisme à l'origine du nazisme ne diffère nullement de celui du génocide. Il n'est point improbable de rapprocher ces deux événements qui relèvent tout simplement de la bêtise humaine. Philippe Adrien, dans la préface de l'œuvre, le souligne en rendant hommage à l'auteur qui a osé « faire place à la cruauté, cette part maudite du genre humain $^{10} \gg$. Nazisme ou génocide rwandais, c'est le genre humain qui est la victime de l'homme. L'un peut donc s'éclairer à la lumière de l'autre. La couleur ou le territoire importe moins que l'être. Toute distinction ou différenciation relève du déni. Il n'y a pas des humanités mais l'Humanité. Nicolas y souscrit dans Nègrerrances :

\section{LE BALAYEUR}

Le mensonge te va si bien. Pourquoi tu ne me parles-tu pas de cet autre frère, le vrai, ce frère jumeau élevé dans l'opulence et l'insouciance ? Cet autre toi-même qui a toujours raison à ses yeux, qui commande et qui ordonne? Ce double de toi éduqué dans l'amour exclusif de soi et le mépris de l'autre?

\section{NICOLAS}

Nous sommes tous pareils, tous égaux, noirs, blancs...

\section{LE BALAYEUR}

Menteur! Pour ce frère, les uns ont le rythme dans la peau, ils parlent trop fort, ils rient trop fort et ils ne sont bons à rien; les autres sont flegmatiques, intelligents, raffinés et ils puent le camembert.

\section{NICOLAS}

Mensonge! La nature est bonne et nous sommes tous frères...

(Nègrerrances, II, p. 45) 
La race, la couleur ne peuvent constituer un critère de hiérarchisation des hommes. Elles contribuent à la splendeur de l'humanité. Contre les apparences, l'être humain est partout le même. La différence de couleur participe du refus de la monotonie dans la création. L'homme transcende la chromaticité.

\section{L'art de la dislocation des frontières génériques}

Dans la tradition classique, au sens de ce qui est institué, préconisé, chaque genre vaut par et pour lui-même; d'où l'existence de nomes, de conventions. Toute transgression des codes d'un genre relève alors de l'inesthétique. Ce pacte de lecture ou d'écriture ne convient pas à José Pliya. La composition de ses œuvres promeut le décloisonnement des genres. Les constantes normatives du genre dramatique ne sont pas toujours avalisées.

\section{Les stratégies du dialogisme artistique}

La mise en crise des certitudes du lecteur-spectateur se formule avec l'épigraphe de Nègrerrances qui est une citation tirée de la chanson I need you turn to (1970) d'Elton John :

And I wonder sometimes and I know I'm unkind

But I need you to turn to when I act so blind

And I need you to turn to when I lose control

You're my guardian angel who keeps out the cold

(Nègrerrances, p. 13)

En substance, on a besoin de l'autre pour être meilleur. Autant les hommes ont besoin les uns les autres, autant la dynamique des arts induit qu' ils interpénètrent, qu'ils ne s'excluent pas.

La suppression des frontières artistiques s'invite ainsi dans cette épigraphe. En faisant de paroles de chanson la légitimation de sa vision du monde, Pliya atteste que les arts peuvent coexister sans se dissoudre les uns dans les autres. Cette forme d'écriture de la porosité des frontières des arts se caractérise par l'intrusion d'un art dans un autre art. Il s'agit d'une présence résiduelle de sorte que l'art qui emprunte préserve sa poétique, son mode d'énonciation, ses codes et ses conventions. 
L'automne déjà ! Mais pourquoi regretter un éternel soleil, si nous sommes engagés à la découverte de la clarté divine, loin des gens qui meurent sur les saisons.

L'automne. Notre barque élevée dans les brumes immobiles tourne vers le pont de la misère, la cité énorme au ciel taché de feu et de boue. Ah! Les haillons pourris, le pain trempé de pluie, l'ivresse, les mille amours qui m'ont crucifié! Elle ne finira donc point cette goule reine de millions d'âmes et de corps morts et qui seront jugés! Je me revois la peau rongée par la boue et la peste, des vers plein les cheveux et les aisselles et encore de plus gros vers dans le cour, étendu parmi les inconnus sans âge, sans sentiment... J'aurais pu y mourir... L'affreuse évocation! J'exècre la misère.

Et je redoute l'hiver parce que c'est la saison du confort.

(Il arrête de dire le poème, s'immobilise et sortant une noix de sa poche, il la mâche.) (Nègrerrances, I, p. 17)

La didascalie «il arrête de dire le poème » admet une altérité artistique à l'œuvre par l'entremise de la marque essentielle du discours direct, les guillemets. La présence de celles-ci crée une sorte de mise en abyme de la poésie dans le théâtre. Par le truchement de la déclamation, le personnage Le Balayeur se fait poète et instaure une interaction artistique qui fissure les cloisons des genres. Tout comme l'intertextualité, l'emboîtement générique confirme le franchissement des frontières par José Pliya, avec une forte influence de Rimbaud :

Les références à la poésie rimbaldienne sont nombreuses. [...] Le début du poème «Adieu » extrait d'Une saison en enfer est récité par le balayeur à l'ouverture de Nègrerrances; la célèbre phrase qui clôt le poème en prose «conte » est aussi mise en exergue de Quêtes : « la musique savante manque à notre désir $\gg^{11}$.

Initiée dans Nègrerrances, l'intermedia artistique se retrouve également dans Pudeur ${ }^{12}$. Pour évoquer les heurs et malheurs de la pratique échangiste, l'auteur emprunte à l'univers pornographique certaines vedettes pour en faire ses personnages. Ce sont Rocco, Tabatah, Ovidie, etc. La pièce en devient une mise en parole de l'action pornographique manifeste dans la truculence des propos de personnages exempts de tabous langagiers :

[...] J'étais mal, calée, blottie entre ces bras qui me parcouraient, qui me fouillaient en toute transparence. J'étais mal. On s'en fait une montagne, mais ce n'est 
rien. Juste un corps étranger qui s'agrippe à vous pleins d'intentions viriles et se laisser faire, se laisser prendre, porter, soulever par cet inconnu aux mains larges, au sexe énorme qui ne demande qu'à vous pénétrer. (Pudeur, p. 73)

Avec cette interartialité, la bienséance instituée par la dramaturgie classique devient vaine tentative de censurer la liberté d'expression dans l'art.

\section{Le travestissement du genre dramatique}

La forme achevée de la dissolution des frontières génériques s'observe dans Lettres à l'humanité avec l'avènement du théâtre épistolaire. Alors qu'il avait en projet une pièce autour des «Noirs dans les camps nazis », les contraintes d'une demande du quotidien l'Humanité l'obligent à « choisir la « lettre-monologue » et à élargir les épistoliers (pas uniquement des Noirs) et les époques (de la Première Guerre mondiale à la fondation d'Israël) ${ }^{13} \gg$. Après six premières lettres, et sur commande de la metteure en scène Sophie Akrich, quatre autres lettres permettent de passer de la fondation d'Israël à l'actualité. Ce qui lui permet de « rajouter les voix d'autres exclus : les pieds noirs, les jeunes des banlieues parisiennes, les sans-papiers ${ }^{14} \gg$. Suivant cette forme épistolaire, la discontinuité dramatique et l'interaction dramatique différée caractérisent la pièce. La prédominance de la fonction phatique signifie le caractère épistolaire de l'œuvre : «Monsieur le Marechal » (Lettre 1, p. 7), « Monsieur le Juge du Conseil de Guerre » (Lettre 2, p. 11), «Husseina ma chérie » (Lettre 3, p. 15), «Bien à vous » (Lettre 4, p. 19), «Mein Führer $\gg($ Lettre 5, p. 23), « Monsieur le Président Ben Gourion » (Lettre 6, p. 27), «Monsieur le Général » (Lettre 7, p. 31), «Buenos dias Señor Schwarzenegger » (Lettre 8, p. 35), « Monsieur le Président de la République $\gg($ Lettre 9, p. 38) et «Mademoiselle $\gg($ Lettre 10, p. 43). Audelà de la réinvention du monologue, le théâtre épistolaire a l'avantage de peindre des faits sociaux déterminés par l'histoire. Chacune des lettres fonctionne comme un témoignage de sorte à conférer à l'ensemble un certain réalisme, captation de la vérité.

La pièce épistolaire se veut une approche novatrice dans la recherche de la vraisemblance. Par le collage, José Pliya démultiplie des situations qui présentent un monde social plein de désillusion. Les lois du genre théâtral sont contestées et reformulées. Lettres à l'humanité s'apprécie, en effet, comme la compilation de dix œuvres autonomes. Chaque lettre valant par et pour ellemême. Les rapports de forces sociaux ne sont pas mis en scène, encore moins des caractères. Les genres épistolaires et dramatiques fusionnent afin de développer la conscience de l'Histoire à travers plusieurs histoires. 


\section{La mise en œuvre d'un théâtre tout-monde}

Du Bénin à la Guadeloupe, en passant par la France, José Pliya se définit par l'errance qui se moque des frontières, plus artificielles que naturelles, constituées par les hommes. De cette transhumance territoriale, il rassemble en lui diverses cultures qui impactent sa vision du monde et son esthétique théâtrale. Contre le fardeau de l'héritage du père mis en scène dans Konda le roquet, son théâtre va à la rencontre du monde. Il entend être de partout et de nulle part dans la mesure où « le théâtre est une question de rencontres ${ }^{15}$ ». La tentation de l'universel se découvre sous différents traits de sa poétique.

\section{La modalité de l'incontestable impartialité}

Les pièces Parabole ${ }^{16}$ et Quêtes ${ }^{17}$ déploient, éloquemment, l'aspiration à l'universalité de José Pliya. Dans cette perspective, ces propos de L'Amante dans Quêtes édifient quant à la connaissance de la Bible par José Pliya.

L'Amante: $[. .$.$] Comme dans une parabole où le berger confie le troupeau à une$ meute de loups pour aller rattraper la brebis égarée, la fugueuse, la capricieuse qui n'est pas vraiment partie, qui s'est juste cachée du berger pour le taquiner, vérifier combien il tient à elle pour la volupté des retrouvailles. (Quêtes, 4, p. 24)

Ces dires renvoient, dans le Nouveau testament, à l'évangile selon Mathieu, XVIII, v.12-14. Partir permet de tester l'amour de l'autre et de renforcer les liens à la réapparition. Les emprunts au texte biblique sont plus nombreux dans Parabole, pièce inspirée du récit biblique de l'enfant prodige. Ce sont des versets qui servent à définir l'architecture externe de l'œuvre. L'auteur, lui-même en indique les références bibliques :

Un homme avait deux fils. Le plus jeune dit à son père : «Père, donne-moi la part d'héritage qui me revient $\gg$. Et le père fit le partage de ses biens. Peu de jours après, le plus jeune rassembla tout ce qu'il avait et partit pour un pays lointain. Lc, XV, 11-13 (Parabole, p. 7)

Il envoie encore d'autres serviteurs dire : «Voilà mon repas est prêt, mes boufs et mes bêtes grasses sont égorgés, tout est prêt. » Mais, il n'en tient pas compte et s'en va au champ. Mt XXII, 4-5 (Parabole, p. 11)

L'artisan sur fer appointe un burin, le passe dans les braises, le façonne au marteau, le travaille d'un bras énergique. Is, XLIV, 12. (Parabole, p. 21)

Celui qui entre par la porte c'est lui, le berger des brebis. Ses brebis à lui, il les appelle chacune par son nom et il les fait sortir. Il marche à leur tête et elles le suivent car elles connaissent sa voix. Jamais elles ne suivent un inconnu car elles ne connaissent pas la voix des inconnus. Jn, X, 2-5 (Parabole, p. 25) 
Un homme a semé du bon grain dans son champ de blé. Or pendant qu'il dormait, son ennemi survient, sème de l'ivraie au milieu du blé et s'en va. Mt, XIII, 24-25 (Parabole, p. 29)

Un homme planta une vigne, il l'entoura d'une clôture, $y$ creusa un pressoir et $y$ bâtit une tour de garde. Mc, XII, 1 (Parabole, p. 39)

« Où est ton frère?

- Je ne sais. Suis-je le gardien de mon frère?»

Gn, IV, 9 (Parabole, p. 49)

Par ce procédé de l'intertextualité, Pliya réattribue à ses textes l'autorité et l'universalité de l'hypertexte biblique. Son théâtre concerne et engage l'humanité entière puisque la Bible se reconnaît à sa supra territorialité. L'impartialité universelle du discours biblique est transférée au théâtre de Pliya devenu ainsi impersonnel.

\section{L'écriture d'une individualité imprécise}

L'indétermination figure parmi les caractéristiques poétiques du théâtre de Pliya. L'identité des personnages demeure indéfinie, car désignés par des noms communs plus aptes à exprimer l'aspiration à l'universel de l'auteur. Ils tendent à définir le rôle des personnages, en insistant sur le lien de parenté. Ainsi dans Parabole, il est à noter la présence du Père, de L'Aîné et du Cadet. L'individu s'efface au profit de la famille dont la pièce évoque les dissensions, les déchirements. Générique, la dénomination participe du refus de l'ancrage territorial et racial. Par l'indifférenciation identitaire, l'auteur parvient à indexer tout le monde et personne. La situation l'emporte alors sur le caractère induit dans un nom propre, marque de l'individuation. Les noms propres sont des marqueurs sociaux à même de favoriser quelque discrimination identitaire. Ce type de nomination réussit à réaliser la distanciation brechtienne. Tout le monde est concerné sans que personne ne soit visé en particulier. L'esthétique de Pliya en devient impressionniste.

Quêtes perpétue la poétique de la dilution de l'identité avec le système de binôme antonymique «La femme/La maîtresse », «Le mari/L'Lamant », pour parachever avec le couple «L'épouse/L'époux ». Cette configuration du système s'organise autour d'une sorte d'opposition entre le pur et l'impur, la morale et l'immoral afin de traduire les faussetés et le mensonge qui affectent les relations humaines. L'hypocrisie et l'insincérité menacent les couples, les fragilisent et les brisent. Par les noms communs, l'auteur révèle que l'infidélité est indissociable de la fidélité. Le statut matrimonial se fait 
dérisoire, incertain à cause du jeu de rôle inféré dans le système du double inhérent au binôme.

Il arrive, aussi, que les personnages soient désignés par le genre et le lien social. Dans Nous étions assis sur le rivage du monde... «La Femme », «L'Homme », « L'Ami », «L'Amie » occupent l'espace dramatique. Ici, la guerre des genres sous-tendue par la présence de L'homme et de La femme se change en opposition des races. Il revient à L'ami et L'amie de réconcilier les antagonistes. La nomination autorise la critique sans le parti pris d'une onomastique déterminant une société donnée. La critique des stéréotypes et des clichés racistes en est plus ouverte. L'imprécision de la désignation énonce que tous les hommes sont susceptibles d'être victimes et auteurs de racisme. «Le Balayeur » de Nègrerrances indique que dans les sociétés actuelles, la fonction revêt une importance inouie. La profession détermine l'être de l'individu qui ne vaut que par l'activité qu'il exerce. Une critique des dérives du capitalisme se ressent en filigrane dans cette prévalence de l'essence sur l'existence.

L'effacement de l'identité des personnages surmonte le confinement de l'œuvre de Pliya dans une sphère sociale définie. Désigner au lieu de nommer sert à peindre des personnages représentatifs de l'universel. José Pliya luimême reconnaît : «Je ne me revendique pas de pouvoir parler d'une couleur, d'un pays mais de parler de l'être humain, de l'homme tout simplement » et d'ajouter «Jamais les personnages ne mettent en avant leur couleur [... J'écris rarement des personnages en pensant à une couleur ou une origine ${ }^{18} \gg$. En un mot, José Pliya estime qu'il faut dépasser les particularismes réducteurs et inhibiteurs.

\section{Conclusion}

Suivant divers procédés, la traversée des frontières sous-tend l'écriture dramatique de José Pliya à travers les pièces évoquées. Les arts et les genres s'interpénètrent pour décliner l'impropriété de leur séparation héritée du classicisme. Il prend à rebrousse-poil les certitudes esthétiques du lecteur-spectateur.

Le théâtre de Jose Pliya n'offre pas de dénouement, de résolution de conclusion par plus de certitudes. L'auteur joue avec les conventions dramaturgiques aristotéliciennes, détourne les règles en faisant commencer ses pièces par la fin et en les faisant se terminer par un nouveau début. Cette inversion de la structure dramatique traditionnelle n'est pas un pur jeu formel mais prouve le refus de l'immobilité et de la fixité qui, sont synonymes de la mort pour Pliya ${ }^{19}$. 
Dans ce contexte, écrire pour un peuple, une race parait insensé. Ses modèles sont souvent étrangers à ses origines africaines. Ainsi, la problématique des frontières des littératures et des arts s'inscrit, lui, dans un processus de renouvellement de l'esthétique dramatique négro-africaine sclérosée par un complexe d'infériorité qui oblige à une réhabilitation de la culture et de l'histoire africaines. Le théâtre de Pliya informe d'une certaine communauté de bien historique et culturelle à ne pas fragmenter au risque de compromettre le devenir même de l'Humanité. Il est impossible de se penser soi sans l'autre. Sans l'autre, rien n'est. D'où l'impossible stratification raciale et artistique défendue par José Pliya en quête d'une fratrie du multiple unificateur et non pas ségrégationniste.

\section{Bibliographie}

Bayart Jean-François, L'illusion identitaire, Paris, Fayard, 1996.

Bérard Stéphanie, Le théâtre-monde de José Pliya, Paris, Honoré Champion, 2015.

Chalaye Sylvie, «Entretien avec José Pliya », in Théâtre/Public, n 158, 2001.

- Afrique noire et dramaturgies contemporaines : le syndrome Frankenstein, Paris, Éditions Théâtrales, 2004.

Conesa Pierre, La fabrication de l'ennemi ou comment tuer avec la conscience pour soi,

Paris, Robert Laffont, « Le monde comme il va », 2011.

Fromilhague Catherine, Les figures de style ( $2^{\mathrm{e}}$ édition), Paris, Armand Colin, 2010. Genette Gérard, Théories des genres, Paris, Seuil, 1985.

Pliya José, Nègrerrances suivi de Konda le Roquet et de Concours de circonstances, Paris, L'Harmattan, 1997.

- Le complexe de Thénardier, Paris, L'avant-scène théâtre/Collection des Quatre-Vents, 2001.

- Une famille ordinaire, Paris, L'avant-scène théâtre/Collection des QuatreVents, 2002.

— , Parabole, Paris, L'Avant-scène théâtre/Collection des Quatre-Vents, 2003.

— Pudeur précédé de Cannibales, Paris, L'avant-scène théâtre/Collection des Quatre-Vents, 2004.

—_, Nous étions assis sur les rivages du monde..., Paris, L'Avant-scène théâtre/ Collection des Quatre-Vents, 2004.

—, Quêtes, Paris, L'Avant-Scène théâtre/Collection des Quatre-Vents, 2005.

—, Lettres à l'bumanité, Carnières-Morlanwelz, Lansman, 2008.

Sarfati Georges-Élia, Éléments d'analyse du discours, Paris, Armand Colin, 2005. 
La crise de la doxa esthétique ou contre les frontières...

\section{Notes}

1. Stéphanie Bérard, Le théâtre-monde de José Pliya, Paris, Honoré Champion, 2015, p. 30.

2. Ibid., p. 20.

3. Catherine Fromilhague, Les figures de style, Paris, Armand Colin, 2010, p. 23.

4. Jean-François Bayart, L'illusion identitaire, Paris, Fayard, 1996, p. 177.

5. Pierre Conesa, La fabrication de l'ennemi, Paris, Robert Laffont, 2011, p. 38.

6. Gérard Genette, «L'Larchitexte », in Théories des genres, Paris, Seuil, 1985, p. 7.

7. José Pliya, Le complexe de Thénardier, Paris, L'avant-scène théâtre/Collection des Quatre-Vents, 2001.

8. Georges-Élia Sarfati, Éléments d'analyse du discours, Paris, Armand Colin, 2005, p. 53.

9. José Pliya, Une famille ordinaire, Paris, L'avant-scène théâtre/Collection des QuatreVents, 2002.

10. Ibid., p. 7.

11. Stéphanie Bérard, op. cit., p. 25.

12. José Pliya, Pudeur, Paris, L'Avant-scène théâtre/Collection des Quatre-Vents, 2004.

13. José Pliya, Lettres à l'humanité, Carnières-Morlanwelz, Lansman, 2008, p. 5.

14. Ibid., p. 6.

15. Sylvie Chalaye, «Entretien avec José Pliya », Théâtre/Public, n 158, 2001, p. 76.

16. José Pliya, Parabole, Paris, L'Avant-scène théâtre/Collection des Quatre-Vents, 2003.

17. José Pliya, Quêtes, Paris, L'Avant-Scène théâtre/Collection des Quatre-Vents, 2005.

18. Sylvie Chalaye, "José Pliya : inventer sa langue " (entretien), in Afrique noire et dramaturgies contemporaines : le syndrome Frankenstein, Paris, Éditions Théâtrales, 2004, p. 94.

19. Stéphanie Bérard, op. cit., p. 8. 